\title{
Brief Research Communication Failure to Replicate Effect of Kibra on Human Memory in Two Large Cohorts of European Origin
}

\author{
Anna C. Need, ${ }^{1}$ Deborah K. Attix, ${ }^{2}$ Jill M. McEvoy, ${ }^{1}$ Elizabeth T. Cirulli, ${ }^{1}$ Kristen N. Linney, ${ }^{1}$ \\ Ana Patricia Wagoner, ${ }^{3}$ Curtis E. Gumbs, ${ }^{1}$ Ina Giegling, ${ }^{4}$ Hans-Jürgen Möller, ${ }^{4}$ Clyde Francks, ${ }^{5}$ \\ Pierandrea Muglia, ${ }^{5}$ Allen Roses, ${ }^{5}$ Greg Gibson, ${ }^{3}$ Mike E. Weale, ${ }^{1}$ Dan Rujescu, ${ }^{4}$ and David B. Goldstein ${ }^{1 *}$ \\ ${ }^{1}$ Institute for Genome Sciences and Policy, Center for Population Genomics and Pharmacogenetics, Duke University, \\ Durham, North Carolina \\ ${ }^{2}$ Division of Neurology and Division of Medical Psychology, Duke University Medical Center, Durham, North Carolina \\ ${ }^{3}$ Department of Genetics, Gardner Hall, North Carolina State University, Raleigh, North Carolina \\ ${ }^{4}$ Department of Psychiatry, Ludwig-Maximilians University, Munich, Germany \\ ${ }^{5}$ GlaxoSmithKline, RTP, North Carolina
}

\begin{abstract}
It was recently suggested that the Kibra polymorphism rs17070145 has a strong effect on multiple episodic memory tasks in humans. We attempted to replicate this using two cohorts of European genetic origin $(n=319$ and $n=365)$. We found no association with either the original SNP or a set of tagging SNPs in the Kibra gene with multiple verbal memory tasks, including one that was an exact replication (Auditory Verbal Learning Task, AVLT). These results suggest that Kibra does not have a strong and general effect on human memory. (c) 2008 Wiley-Liss, Inc.
\end{abstract}

KEY WORDS: neurocognition; phenotype; cognitive task; replication; neurogenetics

Please cite this article as follows: Need AC, Attix DK, McEvoy JM, Cirulli ET, Linney KN, Wagoner AP, Gumbs CE, Giegling I, Möller H-J, Francks C, Muglia P, Roses A, Gibson G, Weale ME, Rujescu D, Goldstein DB. 2008. Failure to Replicate Effect of Kibra on Human Memory in Two Large Cohorts of European Origin. Am J Med Genet Part B 147B:667-668.

Although large-scale genetic association studies have been largely concentrated on disease endpoints there is growing interest in the study of both intermediate phenotypes of disease [Bearden and Freimer, 2006; Braff et al., 2006] and normal variation. In this context the study of human memory stands out as presenting both exceptional opportunities and exceptional challenges. Human memory abilities vary widely among individuals and have often been shown to have high heritabilities [Thapar et al., 1994; Alarcon et al., 1998; Ando et al., 2001]. Moreover, disorders involving impairment of

This article contains supplementary material, which may be viewed at the American Journal of Medical Genetics website at http://www.interscience.wiley.com/jpages/1552-4841/suppmat/ index.html.

*Correspondence to: David B. Goldstein, Institute for Genome Sciences and Policy, Center for Population Genomics and Pharmacogenetics, Duke University, Durham, NC 27710.

E-mail: d.goldstein@duke.edu

Received 4 April 2007; Accepted 25 September 2007

DOI 10.1002/ajmg.b.30658 memory and other cognitive traits are very poorly treated by available medicines, illustrating the need for the identification of new potential therapeutic targets.

Recently, in the first genome-wide association study to identify memory-related genetic variation, [Papassotiropoulos et al., 2006] showed that an intronic polymorphism (rs17070145) in the brain-expressed gene Kibra associates with recall of a list of 30 words after $5 \mathrm{~min}$ and $24 \mathrm{hr}$ in a Swiss cohort $(\mathrm{n}=341)$. Their first replication study in an US sample $(\mathrm{n}=256)$ showed association of the SNP with the Auditory Verbal Learning Test (AVLT) measuring recall after $30 \mathrm{~min}$ (but not $5 \mathrm{~min}$ ), and the Buschke's Selective Reminding test. Finally, they replicated the finding in 424 young Swiss subjects using recall of semantically unrelated pictures after $10 \mathrm{~min}$. From these results, the authors postulated that the Kibra SNP shows an association with multiple memory tasks. We therefore attempted to replicate this finding using multiple memory tests, including, critically, an exact replication of one of their findings. We use two separate cohorts-the Duke Genetics of Memory cohort $(\mathrm{n}=319)$ and a German cohort $(\mathrm{n}=365)$.

Story recall and list learning in the duke genetics of memory cohort. Following Papassotiropoulos et al., we included rs17070145 genotype (combining the CC and CT genotypes), education, gender and age in a regression model to predict verbal recall memory [Green, 2005] (VRM, see supplementary methods), and found no association $(P=0.63$, $\mathrm{n}=300$ ). As part of a whole-genome study, we also genotyped 79 tagging SNPs in and up to $20 \mathrm{~kb}$ upstream of the Kibra gene. None of these SNPs showed association with verbal recall (lowest uncorrected $P=0.13, \mathrm{n}=319$ ). We went on to check the association of rs 17070145 with a story recall task in a subset of the sample $(\mathrm{n}=76)$ and again we found no effect on either immediate $(P=0.39)$ or delayed recall $(0.39)$.

List learning in the german cohort. The tests described above fail to show any effect of Kibra on two different measures of episodic memory in the Duke sample but do not constitute an exact replication of the original findings. We therefore also considered the German cohort in which the same list learning task (AVLT) was used (see supplementary methods). We checked for association both with rs17070145 itself and with 39 Kibra SNPs from the HumanHap 300 genotyping panel. There was no association between rs17070145 and either "immediate": $(P=0.84)$ or delayed (30 min; $P=0.56$ ) recall in the AVLT task. One SNP from the tagging panel associated with AVLT delay score at $P=0.03$, uncorrected for multiple testing. This SNP was not associated with $\mathrm{rs} 17070145\left(\mathrm{r}^{2}=0.005\right)$.

From Papassotiropoulos et al. we can infer that the genotype explains $6.2 \%$ of total variation for the 5 -min delayed verbal 
recall in Swiss cohort 1 , and $4.1 \%$ for the 30 -min delayed VLMT in the US cohort. We note that our Genetics of Memory cohort was $90 \%$ powered to detect an effect size of $\geq 3.2 \%$ at a significance of 0.05 , and the German cohort was $90 \%$ powered to detect an effect size of $\geq 3.0 \%$. Any effect size greater than $1.7 \%$ should have produced a $P$-value less than the observed one with a probability of at least $95 \%$.

In conclusion, we failed to find an association between Kibra and memory performance in multiple memory tasks, one of which constituted an exact replication. Additionally, it does not appear that differences in the linkage disequilibrium structure between our cohorts was masking a true association with an unidentified functional Kibra variant since we failed to find any association with a large number of tagging SNPs distributed throughout the Kibra gene in either cohort.

This discrepancy highlights the challenges inherent in large scale genetic studies of human memory. It is reasonable to assume that polymorphisms may affect multiple forms of memory, but the rich assortment of cognitive measures, each with multiple indices, can create challenges for consistency across replication efforts. Ideally, the original association should be exactly replicated before exploring genetic effects on related cognitive tasks. These results argue against a strong and general effect of Kibra on episodic memory and highlight the critical need for large and consistently phenotyped cohorts to explore the relationship between genetic variation and human cognition.

\section{REFERENCES}

Alarcon M, Plomin R, Fulker D, Corley R, DeFries J. 1998. Multivariate path analysis of specific cognitive abilities data at 12 years of age in the Colorado Adoption Project. Behav Genet 28:255-264.

Ando J, Ono Y, Wright M. 2001. Genetic structure of spatial and verbal working memory. Behav Genet 31:615-624.

Bearden C, Freimer N. 2006. Endophenotypes for psychiatric disorders: Ready for primetime? Trends Genet 22(6):306-313.

Braff D, Freedman R, Schork N, Gottesman I. 2006. Deconstructing schizophrenia: An overview of the use of endophenotypes in order to understand a complex disorder. Schizophr Bull 33(1):21-32.

Green P. 2005. Story recall test. Edmonton, CA: Green's Publishing.

Papassotiropoulos A, Stephan D, Huentelman M, Hoerndli F, Craig D, Pearson J, Huynh K, Brunner F, Corneveaux J, Osborne D, Wollmer M, Aerni A, Coluccia D, Hanggi J, Mondadori C, Buchmann A, Reiman E, Caselli R, Henke K, de Quervain D. 2006. Common Kibra alleles are associated with human memory performance. Science 314(5798):475-478.

Thapar A, Petrill S, Thompson L. 1994. The heritability of memory in the Western Reserve Twin Project. Behav Genet 24:155-160. 